\title{
Prenatal Nicotine Exposure Recruits an Excitatory Pathway to Brainstem Parasympathetic Cardioinhibitory Neurons during Hypoxia/Hypercapnia in the Rat: Implications for Sudden Infant Death Syndrome
}

\author{
ZHENG-GUI HUANG, XIN WANG, OLGA DERGACHEVA, AND DAVID MENDELOWITZ \\ Department of Pharmacology and Physiology, The George Washington University, Washington, DC 20037
}

\begin{abstract}
ABST
Maternal cigarette smoking and prenatal nicotine exposure
increase the risk for sudden infant death syndrome (SIDS) by 2-
to 4-fold, yet despite adverse publicity, nearly one of four
pregnant women smoke tobacco. Infants who succumb to SIDS
typically experience a severe bradycardia that precedes or is
accompanied by centrally mediated life-threatening apneas and
gasping. Although the causes of the apnea and bradycardia
prevalent in SIDS victims are unknown, it has been hypothesized
that these fatal events are exaggerated cardiorespiratory re-
sponses to hypoxia or hypercapnia. Changes in heart rate are
primarily determined by the activity of cardiac vagal neurons
(CVNs) in the brainstem. In this study, we tested whether
hypoxia/hypercapnia evokes synaptic pathways to CVNs and
whether these cardiorespiratory interactions are altered by pre-
natal exposure to nicotine. Spontaneous rhythmic inspiratory-
related activity was recorded from the hypoglossal rootlet of 700-
to 800- $\mu$ m medullary sections. CVNs were identified in this
\end{abstract}
There are at least two major physiologic interactions between the respiratory system and the neural control of heart rate. The most ubiquitous cardiorespiratory interaction is respiratory sinus arrhythmia. During each respiratory cycle, the heart beat slows during postinspiration/expiration, and heart rate increases during inspiration. Such increases in heart rate during inspiration are observed in a wide variety of mammals, including both neonatal (1) and adult humans (2) and rats (3). Respiratory sinus arrhythmia helps match pulmonary blood flow to lung inflation and maintain the appropriate diffusion gradient for oxygen in the lungs (4).

Received October 28, 2004; accepted January 26, 2005.

Correspondence: David Mendelowitz, Ph.D., Department of Pharmacology and Physiology, The George Washington University, 2300 Eye St., NW, Washington, DC 20037; e-mail: dmendel@gwu.edu.

This work was supported by National Institutes of Health grants HL72006 and HL59895 to D.M.

DOI: 10.1203/01.PDR.0000179380.41355.FC preparation by retrograde fluorescent labeling, and excitatory synaptic inputs to CVNs were isolated and studied using patchclamp electrophysiologic techniques. Hypoxia/hypercapnia did not elicit an increase in excitatory neurotransmission to CVNs in unexposed animals, but in animals that were exposed to nicotine in the prenatal period, hypoxia/hypercapnia recruited an excitatory neurotransmission to CVNs. This study establishes a likely neurochemical mechanism for the exaggerated decrease in heart rate in response to hypoxia/hypercapnia that occurs in SIDS victims. (Pediatr Res 58: 562-567, 2005)
Abbreviations
aCSF, artificial cerebrospinal fluid
CVN, cardiac vagal neuron
NA, nucleus ambiguus
SIDS, sudden infant death syndrome

Although feedback from pulmonary stretch receptors, direct respiratory related-changes in venous return, and cardiac stretch can evoke respiratory-related fluctuations in heart rate, the dominant source of respiratory sinus arrhythmia originates from the brainstem $(4,5)$. Respiratory sinus arrhythmia persists when the lungs are stationary (caused by muscle paralysis or constant flow ventilation), and the respiratory modulation of heart rate remains synchronized with brainstem respiratory rhythms even when artificial ventilation of the lungs and chemoreceptor activation occur at different intervals (6,7). In animals, including humans, respiratory sinus arrhythmia is mediated via cardiac vagal activity. Respiratory sinus arrhythmia persists in experimental animals upon sectioning sympathetic pathways and in quadriplegic patients with spinal cord injury and sympathetic dysfunction (6-8). Blockade of parasympathetic cardiac activity abolishes respiratory sinus arrhythmia (9). Possible cellular mechanisms of respiratory sinus arrhythmia include activation of excitatory pathways to pre- 
motor cardiac vagal neurons (CVNs) during postinspiration and/or an increased inhibition of premotor CVNs during inspiration.

Among the strongest interactions between the cardiovascular and respiratory systems are the responses to hypoxia and hypercapnia. Hypoxia initially elicits an increase, followed by a dramatic decrease in respiratory frequency (10). In addition, hypoxia transforms eupneic respiratory activity to gasping activity, which is characterized by infrequent, robust breaths of short duration $(11,12)$. Prolonged hypoxia and hypercapnia eventually produce a terminal apnea. Likewise, hypoxia and hypercapnia evoke an initial transient increase in heart rate followed by a parasympathetically mediated bradycardia and, ultimately, cessation of cardiac contractions $(13,14)$. The reduction in heart rate and respiratory frequency in response to hypoxia normally serves to reduce the metabolic demand of the cardiac and respiratory muscles and thus prolong survival (15).

Exaggeration of this protective response to hypoxia, however, could be detrimental. Sudden infant death syndrome (SIDS) is the leading cause of infant death in the postneonatal period (16). Infants who succumb to SIDS typically experience a severe bradycardia that precedes or is accompanied by centrally mediated life-threatening apneas and gasping $(8,10)$. Although the causes of the apnea and bradycardia prevalent in SIDS victims are unknown, it has been hypothesized that these fatal events are exaggerated cardiorespiratory responses to hypoxia or hypercapnia $(17,18)$.

Although the cause(s) for SIDS remains unknown, chronic fetal nicotine exposure by maternal smoking dramatically increases the risk for SIDS. Epidemiologic studies indicate that smoking during pregnancy increases the risk for SIDS by two to four times $(19,20)$. In animal models of SIDS, prenatal nicotine exposure augments the parasympathetic and diminishes the sympathetic control of heart rate (21). Prenatal exposure to nicotine also exaggerates the cardiovascular responses to hypoxia. In control rats, hypoxia typically evokes an initial tachycardia followed by a slight decrease in heart rate, whereas in nicotine-exposed animals, there is no tachycardia and heart rate declines rapidly and precipitously within a few minutes (22).

This study was designed to test the following three hypotheses: 1) premotor CVNs in the nucleus ambiguus (NA) receive rhythmic respiratory-related excitatory synaptic input during the period of postinspiration in eupneic-like respiration; 2) respiratory activity changes to a gasp-like pattern in response to hypoxia/ hypercapnia, which is accompanied by an increased excitatory synaptic neurotransmission to CVNs; and 3) animals that are exposed to nicotine in the prenatal period have exaggerated cardiorespiratory responses to hypoxia/hypercapnia.

\section{METHODS}

Fluorescent labeling of CVNs and medullary slice preparation. Neonatal Sprague-Dawley rats (P3-P7; Hilltop, Scottdale, PA) were anesthetized with ketamine/xylazine $(87 / 13 \mathrm{mg} / \mathrm{kg})$ and cooled to $\sim 4^{\circ} \mathrm{C}$ to slow the heart rate. A right thoracotomy was performed, and the retrograde fluorescent tracer X-rhodamine-5-(and-6)-isothiocyanate (Molecular Probes, Eugene, OR) was injected into the fat pads at the base of the heart. After $24-48 \mathrm{~h}$ of recovery, animals were anesthetized with halothane and killed by cervical dislocation, and the brain tissue was placed in a $4{ }^{\circ} \mathrm{C}$ physiologic saline solution $(140 \mathrm{mM}$
$\mathrm{NaCl}, 5 \mathrm{mM} \mathrm{KCl}, 2 \mathrm{mM} \mathrm{CaCl} 2,5 \mathrm{mM}$ glucose, and $10 \mathrm{mM}$ HEPES) bubbled with $100 \% \quad \mathrm{O}_{2}(\mathrm{pH}$ 7.4). All animal procedures were performed with the approval of the Animal Care and Use Committee of The George Washington University in accordance with the recommendations of the panel on euthanasia of the American Veterinary Medical Association and the National Institutes of Health publication, "Guide for the Care and Use of Laboratory Animals."

The medulla was removed with care to preserve the hypoglossal cranial nerve rootlet. The medulla was mounted on a cutting block and placed into a vibrating blade microtome (Leica, Nussloch, Germany). Serial transverse sections were sliced in a rostrocaudal progression until the inferior olives and the NA could be visualized on the rostral surface of the tissue. A single thick (700-800 $\mu \mathrm{m}$ ) section that included CVNs, the hypoglossal nerve rootlet, the pre-Bötzinger complex, and the rostral portion of the hypoglossal nucleus was cut and submerged in a recording chamber that allowed perfusion $(4 \mathrm{~mL} / \mathrm{min})$ above and below the slice with room temperature artificial cerebrospinal fluid (aCSF; $125 \mathrm{mM} \mathrm{NaCl}, 3 \mathrm{mM} \mathrm{KCl}, 2 \mathrm{mM} \mathrm{CaCl}{ }_{2}, 26 \mathrm{mM} \mathrm{NaHCO}_{3}, 5 \mathrm{mmol}$ glucose, and $5 \mathrm{mM}$ HEPES) equilibrated with $95 \% \mathrm{O}_{2} / 5 \% \mathrm{CO}_{2}(\mathrm{pH} 7.4)$.

Recording respiratory network activity. The thick medullary slice preparation generates rhythmic inspiratory-related motor discharge in hypoglossal cranial nerves (23). Spontaneous respiratory-related activity was recorded by monitoring motor-neuron population activity from hypoglossal nerve rootlets using a suction electrode. Hypoglossal rootlet activity was amplified (50,000 times) and filtered (10- to 300-Hz band pass; CWE Inc., Ardmore, PA) and electronically integrated ( $\tau=50 \mathrm{~ms}$; CWE Inc.).

Patch-clamp techniques. CVNs in the NA were identified by the presence of the fluorescent tracer as described previously (24). Briefly, slices were viewed with infrared illumination and differential interference optics (Zeiss, Oberkochen, Germany) and under fluorescent illumination with an infrared sensitive cooled charged-coupled device camera (Photometrics, Tucson, AZ). Neurons that contained the fluorescent tracer were identified by superimposing the fluorescent and infrared images on a video monitor (Sony, Tokyo, Japan). Patch pipettes (2.5-3.5 M $\Omega$ ) were visually guided to the surface of individual CVNs using differential interference optics and infrared illumination (Zeiss, Oberkochen, Germany). CVNs were voltage-clamped at a holding potential of $-80 \mathrm{mV}$. The patch pipettes were filled with a solution that consisted of (in $\mathrm{mM}) 135 \mathrm{~K}$-gluconic acid, $10 \mathrm{HEPES}, 10 \mathrm{EGTA}, 1 \mathrm{CaCl}_{2}$, and $1 \mathrm{MgCl}_{2}$, at a $\mathrm{pH}$ of 7.35-7.4.

Focal drug application. Focal drug application was performed using a pneumatic picopump pressure delivery system (WPI, Sarasota, FL). Drugs were ejected from a patch pipette positioned within $30 \mu \mathrm{m}$ from the patched CVN. The maximum range of drug application has been previously determined to be $100-120 \mu \mathrm{m}$ downstream from the drug pipette and considerably less behind the drug pipette (25). Glutamatergic neurotransmission was isolated by focal application of strychnine $(1 \mu \mathrm{M})$ and gabazine $(25 \mu \mathrm{M})$ to block glycine and $\mathrm{GABA}_{\mathrm{A}}$ receptors, respectively. All drugs were obtained from Sigma Chemical Co. (St. Louis, MO).

Hypoxia/hypercapnia. Rhythmic inspiratory-related activity and glutamatergic excitatory post-synaptic currents (EPSCs) in a single CVN were recorded simultaneously for $4 \mathrm{~min}$ in aCSF equilibrated with $95 \% \mathrm{O}_{2} / 5 \% \mathrm{CO}_{2}$. Slices then were perfused with hypoxic/hypercapnic aCSF (equilibrated with $85 \% \mathrm{~N}_{2} / 6 \% \mathrm{O}_{2} / 9 \% \mathrm{CO}_{2}$ ) for $10 \mathrm{~min}$ and then returned to the original perfusate for $20 \mathrm{~min}$.

Prenatal nicotine exposure. Adult female rats were anesthetized with ketamine/xylazine $(87 / 13 \mathrm{mg} / \mathrm{kg}$, i.p.; Phoenix Pharmaceuticals, St. Joseph, MO) on the third day of gestation, and Alzet osmotic minipumps (Durect, Cupertino, CA) that contained nicotine $(56.1 \mathrm{mg} / \mathrm{mL}$ bacteriostatic saline; Sigma Chemical Co.) were implanted. Osmotic minipumps were chosen to avoid the high plasma nicotine concentrations and subsequent episodic fetal hypoxia/ischemia that can be produced by nicotine injections. Pumps delivered $2.1 \mathrm{mg}$ nicotine/d, a level approximately equivalent to those that occur in moderate to heavy smokers, for $28 \mathrm{~d}$ (22). Nicotine-treated animals continued to be exposed to nicotine through maternal milk until they were killed.

Data analysis. Synaptic events were detected using MiniAnalysis version 5.6.12 (Synaptosoft, Decatur, GA). The frequency of EPSCs that occurred in CVNs were grouped into 1 -s bins and cross-correlated with the onset of inspiratory-related hypoglossal activity. Data were analyzed from all bursts during the last 2 min of the control period, during the last 2 min of the 10-min period of hypoxia/hypercapnia, and from minutes 10 to 12 during the 20 -min recovery. These periods were chosen for analysis because during these periods, synaptic activities were consistent and any hypoxia/hypercapnia-evoked changes reached a steady state. Burst duration and frequency were measured using pClamp 7 software (Axon Instruments, Union City, CA) from the filtered (10- to $300-\mathrm{Hz}$ band pass) and electronically integrated hypoglossal rootlet activity. Results are presented as mean \pm SEM. Statistical comparisons were performed using ANOVA with repeated measures to examine the responses throughout the time course of the experiments and unpaired $t$ test when 
comparing the results from control animals with animals that were exposed to nicotine prenatally. Significant difference was set at $p<0.05$.

\section{RESULTS}

When the perfusate was changed from aCSF equilibrated with $95 \% \mathrm{O}_{2} / 5 \% \mathrm{CO}_{2}$ to aCSF equilibrated with $85 \% \mathrm{~N}_{2} / 6 \%$ $\mathrm{O}_{2} / 9 \% \mathrm{CO}_{2}$, respiratory frequency initially increased, which was followed by a depression in the frequency of bursting (Fig. 1). Hypoxia/hypercapnia also evoked a significant decrease in the burst duration, as shown in Fig. 1. The respiratory responses in animals that were exposed to nicotine in the prenatal period closely mimicked and were not significantly different from the responses in unexposed animals (Fig. 1).

Glutamatergic synaptic neurotransmission to CVNs in unexposed animals was not modulated by respiratory activity during either control or hypoxia/hypercapnia conditions, as shown in Fig. 2. The frequency of EPSCs in CVNs did not significantly change either during the eupneic-like bursts or
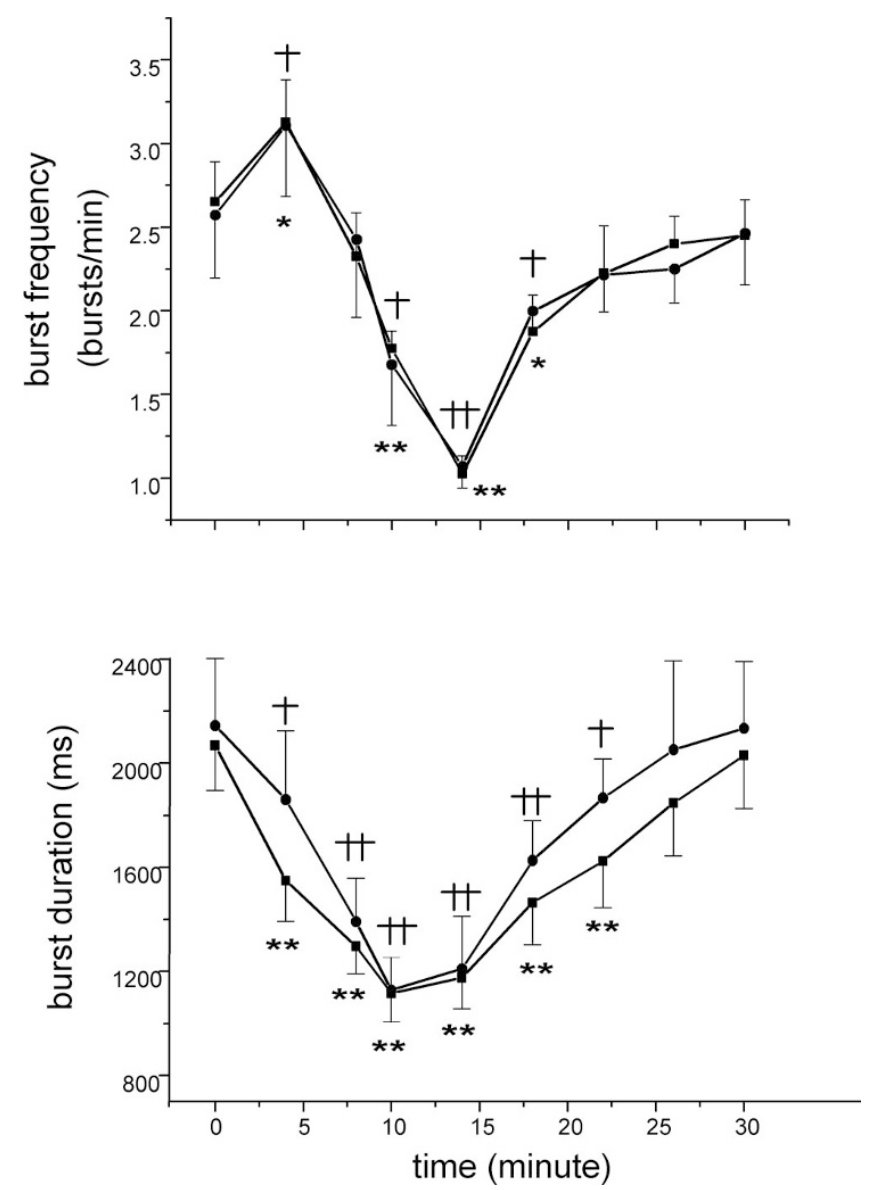

Figure 1. Hypoxia/hypercapnia evokes biphasic changes in respiratory activity. (Top) A change in the perfusate from aCSF equilibrated with $95 \%$ $\begin{array}{llllll}\mathrm{O}_{2} / 5 \% & \mathrm{CO}_{2} & \text { to aCSF equilibrated with } 85 \% & \mathrm{~N}_{2} / 6 \% & \mathrm{O}_{2} / 9 \% & \mathrm{CO}_{2} \text { evoked }\end{array}$ biphasic changes in respiratory activity as burst frequency initially significantly increased then decreased in both unexposed animals $(\boldsymbol{\square})$ and animals that were exposed to nicotine in the prenatal period (-). (Bottom) Hypoxia/ hypercapnia decreased inspiratory burst duration as the bursts became more gasp-like, in both unexposed $(\square)$ and animals that were exposed to nicotine in the prenatal period $(-) . \dagger,{ }^{*} p<0.05, \dagger \dagger,{ }^{*} p<0.01$. The respiratory responses in animals that were exposed to nicotine in the prenatal period were not significantly different from the responses in unexposed animals. during the gasp-like activity present with hypoxia/hypercapnia, either during the bursts or in the postinspiratory phase. Surprisingly, however, in the recovery from hypoxia/hypercapnia, respiratory bursts evoked a significant increase in the frequency of EPSCs in CVNs. As illustrated in a typical experiment (Fig. 2A) and in the average results from 10 neurons (Fig. $2 B$ ), during the recovery from hypoxia/hypercapnia, respiratory bursts elicited an increase in glutamatergic neurotransmission to CVNs.

In animals that were exposed to nicotine throughout the prenatal period, glutamatergic synaptic neurotransmission to CVNs was not modulated by respiratory activity during control conditions, similar to unexposed animals, as shown in Fig. 3. However during hypoxia/hypercapnia, gasp-like respiratory bursts dramatically increased the frequency of EPSCs in CVNs in these prenatal nicotine-exposed animals. Also, in contrast to unexposed animals, there were no significant changes in EPSC frequency in CVNs associated with the respiratory activity in the recovery period after hypoxia/hypercapnia. Results from a typical experiment are shown in Fig. $3 A$, and the average results from eight neurons are shown in Fig. $3 B$.

\section{DISCUSSION}

There are three major results from this study. Premotor CVNs in the NA, in both unexposed and prenatal nicotineexposed animals, did not receive any rhythmic respiratoryrelated excitatory inputs during eupneic-like respiratory activity. Hypercapnic/hypoxic conditions did not elicit any increase

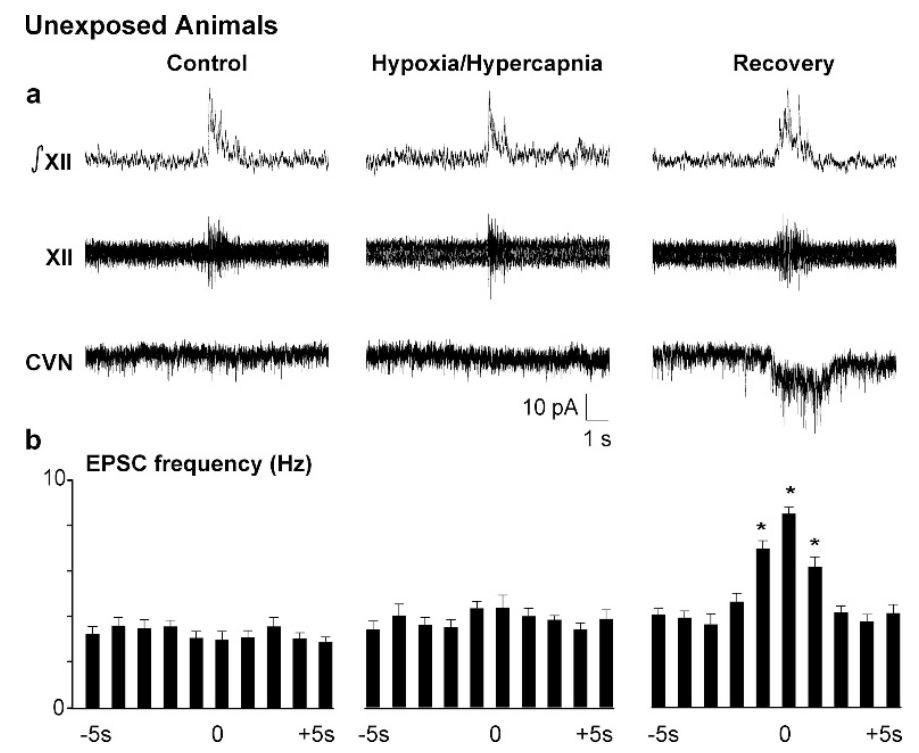

Figure 2. In unexposed animals, there was no excitatory input to CVNs during hypoxia/hypercapnia. Inspiratory-related bursting activity was recorded from the hypoglossal rootlet (XII) and electronically integrated ( $\left.\int \mathrm{XII}\right)$. Fluorescently identified CVNs were patch-clamped in the whole-cell configuration, and glutamatergic neurotransmission was isolated in animals that were unexposed to nicotine. Inspiratory bursts had no effect on the frequency of glutamatergic EPSCs in CVNs during control conditions or during the period of hypoxia/hypercapnia as shown from a typical experiment in $A$ and in the average results from 10 experiments $(B)$. However, during the recovery from hypoxia/hypercapnia, glutamatergic EPSC frequency significantly increased during inspiratory bursts, as shown the typical experiment $(A)$ and the average results from 10 experiments $(B)$. $* p<0.05$. 


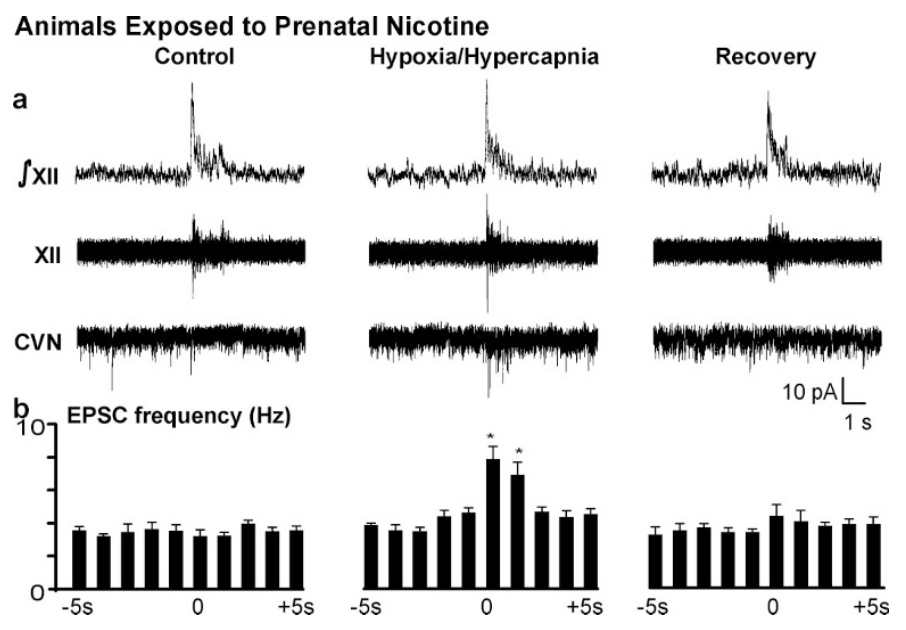

Figure 3. In animals that were exposed to nicotine in the prenatal period, hypoxia/hypercapnia recruits an excitatory pathway to CVNs during gasping. Inspiratory-related bursting activity was recorded from the hypoglossal rootlet (XII) and electronically integrated ( $\left.\int \mathrm{XII}\right)$, whereas glutamatergic EPSCs were simultaneously recorded in CVNs that were isolated from animals that were exposed to nicotine in the prenatal period. Inspiratory bursts had no effect on the frequency of glutamatergic EPSCs in CVNs during the control conditions, as shown from a typical experiment in $A$ and in the average results from eight experiments $(B)$. However hypoxia/hypercapnia recruited an excitatory pathway to CVNs as inspiratory gasps evoked significant increases in excitatory post-synaptic currents (EPSC) frequency during the inspiratory activity. During the recovery from hypoxia/hypercapnia, inspiratory bursts had no effect on the frequency of glutamatergic EPSCs in CVNs from animals that were exposed to nicotine in the prenatal period. $* p<0.05$.

in excitatory neurotransmission to premotor CVNs in unexposed animals, but during recovery from hypoxia/hypercapnia, inspiratory activity evoked an increase in glutamatergic EPSC frequency. In animals that were exposed to nicotine in the prenatal period, hypoxia/hypercapnia recruited a strong excitatory neurotransmission to CVNs during gasp-like respiratory bursts.

Similar to what has been observed from in vivo preparations, hypoxia and/or hypercapnia evoked biphasic changes in respiratory activity as bursting frequency initially increased and subsequently decreased, and burst duration shortened as respiration shifted from an eupneic-like to a gasping-like pattern $(26,27)$. These respiratory responses were not significantly changed by exposure to prenatal nicotine, and the respiratory burst frequency and durations in animals that were exposed to prenatal nicotine were nearly indistinguishable from unexposed animals. The effect of prenatal nicotine exposure on respiratory function in vivo is controversial in both animal models and humans. Prenatal nicotine exposure did not alter the ventilatory response to hypoxia or hypercapnia in rats from 3 to $34 \mathrm{~d}$ of age (28), yet in another study, prenatal nicotine exposure found an attenuation of the biphasic hypoxic ventilatory response in rats (29). In one study, infants of smoking mothers did not have different ventilatory responses to hypoxia or hypercapnia, but these infants did have diminished arousal to hypoxia (30), whereas in another study, infants who were born to smoking mothers had diminished hypoxic ventilatory responses and reduced respiratory drive (31). The results from this study indicate that prenatal nicotine exposure does not alter central hypoglossal respiratory activity and suggests that the variable ventilatory responses to prenatal nicotine exposure in vivo may depend on changes in the activity of peripheral chemoreceptors rather than changes within the brainstem.

It is at first somewhat surprising that premotor CVNs do not receive respiratory-related excitatory neurotransmission in this preparation during any phase of the eupneic-like respiratory cycle given the pronounced respiratory modulation of heart rate. In the only in vivo study in which intracellular recordings were successful (and in only two neurons), excitatory synaptic events in premotor CVNs were present and phasic with the arterial pressure pulse, but the excitatory synaptic events were uncorrelated with the respiratory cycle (32). During inspiration, premotor CVNs were hyperpolarized, and injection of chloride reversed this hyperpolarization, suggesting that a chloride conductance was activated during inspiration (32).

Recent work has characterized the respiratory evoked inputs to CVNs and has demonstrated that respiratory modulation of cardiac vagal activity is mediated by modulation of inhibitory neurotransmission to CVNs. During inspiratory bursts, the frequency of both spontaneous inhibitory GABAergic and spontaneous glycinergic synaptic events in CVNs is significantly increased (33). Focal application of the nicotinic antagonist dihydro- $\beta$-erythroidine to an area immediately surrounding the patched $\mathrm{CVN}$, in an $\alpha 4 \beta 2$ selective concentration (3 $\mu \mathrm{M})$, abolished the respiratory evoked increase in GABAergic frequency (33). In contrast, the increase in glycinergic frequency during inspiration was not altered by nicotinic antagonists.

In animals that were not exposed to nicotine, there was respiratory-related excitation of CVNs not during hypoxia/ hypercapnia but, surprisingly, in the recovery period. This cardiorespiratory interaction may be involved in potentially life-threatening arrhythmias that occur during and after obstructive sleep apnea. Recovery from obstructive sleep apnea in humans occurs either by arousal from sleep or from hypoxic gasping (autoresuscitation). Many patients with sleep apnea syndrome have nocturnal and sometimes severe bradycardia and bradycardic arrhythmias (34). The bradycardia associated with sleep apnea is likely mediated, at least in part, by increases in parasympathetic cardioinhibitory activity because atropine, a blocker of cardiac vagal activity, is partially effective in preventing the majority of arrhythmias during and after obstructive sleep apnea (35). Although the cause of the severe bradycardia associated with sleep apnea is unknown, the results from this study suggest that it may be the result of excitation of parasympathetic cardiac neurons that typically occurs during autoresuscitation (36).

One of the major findings of this study is the demonstration that prenatal nicotine exposure dramatically alters cardiorespiratory interactions in the medulla. Whereas in control animals there was no change in excitatory neurotransmission to CVNs during hypoxia/hypercapnia, in animals that were exposed to nicotine in the prenatal period, hypoxia/hypercapnia recruited a strong excitatory synaptic pathway to CVNs. These results strongly complement recent work that examined whether prenatal nicotine exposure alters inhibitory neurotransmission to CVNs during hyp- 
oxia. In unexposed animals, hypoxia evokes a biphasic change in the frequency of both inhibitory GABAergic and glycinergic synaptic events in CVNs, composed of an initial increase, followed by a decrease in inhibitory post-synaptic current frequency (37). Prenatal exposure to nicotine changed the GABAergic response to hypoxia from a biphasic response to a precipitous decrease in spontaneous GABAergic inhibitory post-synaptic current frequency (37). These results taken together would predict a much stronger excitation of CVNs during hypoxia and/or hypercapnia from animals that were exposed to nicotine in the prenatal period.

The strong excitation of premotor CVNs that occurs in prenatal nicotine animals shown in this study is a strong candidate for mediating cardiovascular dysfunction in SIDS, which is the leading cause of death in infants between $1 \mathrm{mo}$ and $1 \mathrm{y}$ of age. Although the cause(s) for SIDS remains unknown, chronic fetal nicotine exposure by maternal smoking dramatically increases the risk for SIDS by two to four times $(19,20)$. Infants who succumb to SIDS typically have gasp-like respiratory patterns as well as intrathoracic petechial hemorrhages, which are an indication of rigorous respiratory efforts $(36,38)$. Prenatal exposure to nicotine exaggerates the bradycardia response to hypoxia (22), although the mechanisms have not previously been studied. It is likely that recruitment of an excitatory neurotransmission to CVNs during hypoxia/ hypercapnia-evoked gasping, such as that demonstrated in this study in animals that were exposed to nicotine, is responsible for the bradycardia that occurs in infants who succumb to SIDS.

The mechanisms that are responsible for the altered responses with prenatal nicotine exposure likely involve altered neurotransmission within the CNS. Prenatal nicotine exposure has been shown previously to exaggerate the facilitation of glutamatergic neurotransmission to CVNs and also change the types of presynaptic and postsynaptic nicotinic receptors involved in exciting premotor CVNs (39). Changes in the subtype, number, and characteristics of the nicotinic receptors are likely involved. Chronic nicotine exposure has been shown to decrease the number of lowaffinity and increase the number and fraction of high-affinity nicotinic receptors (40). The acetylcholine-evoked ionic currents from these up-regulated nicotinic receptors are augmented 2-fold or more compared with control currents and are less sensitive to desensitization (40).

\section{CONCLUSION}

In summary, during normal eupneic-like respiration, premotor CVNs do not receive any rhythmic respiratory-related excitatory inputs. However, in the recovery period after but not during hypoxia/hypercapnia, there is an increase in excitatory neurotransmission to premotor CVNs in unexposed animals. In animals that were exposed to nicotine in the prenatal period, hypoxia/ hypercapnia recruits a strong excitatory neurotransmission to CVNs during gasp-like respiratory bursts. This study establishes a likely neurochemical mechanism for the heart rate response to hypoxia/hypercapnia and a link between prenatal nicotine expo- sure and an exaggerated bradycardia during hypoxia/hypercapnia that may contribute to SIDS.

\section{REFERENCES}

1. Hathorn MK 1987 Respiratory sinus arrhythmia in new-born infants. J Physiol 385:1-12

2. Hirsch JA, Bishop B 1981 Respiratory sinus arrhythmia in humans: how breathing pattern modulates heart rate. Am J Physiol 241:H620-H629

3. Bouairi E, Neff R, Evans C, Gold A, Andresen MC, Mendelowitz D 2004 Respiratory sinus arrhythmia in freely moving and anesthetized rats. J Appl Physiol 97:1431-1436

4. Anrep G, Pascual W, Rossler R 1936 Respiratory variations of the heart rate. II. The central mechanism of the respiratory sinus arrhythmia and the inter-relations between the central and the reflex mechanisms. Proc R Soc 119:218-232

5. Anrep G, Pascual W, Rossler R 1936 Respiratory variations of the heart rate. I. The mechanism of respiratory arrhythmia reflex. Proc R Soc 119:119-217

6. Daly MD 1991 Some reflex cardioinhibitory responses in the cat and their modulation by central inspiratory neuronal activity. J Physiol 439:559-577

7. Shykoff BE, Naqvi SS, Menon AS, Slutsky AS 1991 Respiratory sinus arrhythmia in dogs. Effects of phasic afferents and chemostimulation. J Clin Invest 87:1621-1627

8. Inoue K, Miyake S, Kumashiro M, Ogata H, Yoshimura O 1990 Power spectral analysis of heart rate variability in traumatic quadriplegic humans. Am J Physiol 258:H1722-H1726

9. Warner MR, deTarnowsky JM, Whitson CC, Loeb JM 1986 Beat-by-beat modulation of AV conduction. II. Autonomic neural mechanisms. Am J Physiol 251:H1134-H1142

10. Guntheroth WG, Kawabori I 1975 Hypoxic apnea and gasping. J Clin Invest 56:1371-1377

11. Gozal D, Gozal E, Reeves SR, Lipton AJ 2002 Gasping and autoresuscitation in the developing rat: effect of antecedent intermittent hypoxia. J Appl Physiol 92:1141-1144

12. Guntheroth WG, Kawabori I 1975 Hypoxia apnea and gasping. J Clin Invest 56:1371-1377

13. Deshpande P, Khurana A, Hansen P, Wilkins D, Thach BT 1999 Failure of autoresuscitation in weanling mice: significance of cardiac glycogen and heart rate regulation. J Appl Physiol 87:203-210

14. Schuen JN, Bamford OS, Carroll JL 1997 The cardiorespiratory response to anoxia: normal development and the effect of nicotine. Respir Physiol 109:231-239

15. Schuen JN, Bamford OS, Carroll JL 1997 The cardiorespiratory response to anoxia: normal development and the effect of nicotine. Respir Physiol 109:231-239

16. Anderson RN 2002 Deaths: leading causes for 2000. Natl Vital Stat Rep 50:1-85

17. Hunt CE 2001 Sudden infant death syndrome and other causes of infant mortality: diagnosis, mechanisms, and risk for recurrence in siblings. Am J Respir Crit Care Med 164:346-357

18. Meny RG, Carroll JL, Carbone MT, Kelly DH 1994 Cardiorespiratory recordings from infants dying suddenly and unexpectedly at home. Pediatrics 93:44-49

19. Mitchell EA, Ford RP, Stewart AW, Taylor BJ, Becroft DM, Thompson JM, Scragg R, Hassall IB, Barry DM, Allen EM, et al 1993 Smoking and the sudden infant death syndrome. Pediatrics 91:893-896

20. Haglund B, Cnattingius S 1990 Cigarette smoking as a risk factor for sudden infant death syndrome: a population-based study. Am J Public Health 80:29-32

21. Slotkin TA, Epps TA, Stenger ML, Sawyer KJ, Seidler FJ 1999 Cholinergic receptors in heart and brainstem of rats exposed to nicotine during development: implications for hypoxia tolerance and perinatal mortality. Brain Res Dev Brain Res 113:1-12

22. Slotkin TA, Saleh JL, McCook EC, Seidler FJ 1997 Impaired cardiac function during postnatal hypoxia in rats exposed to nicotine prenatally: implications for perinatal morbidity and mortality, and for sudden infant death syndrome. Teratology 55: $177-184$

23. Smith JC, Ellenberger HH, Ballanyi K, Richter DW, Feldman JL 1991 Pre-Botzinger complex: a brainstem region that may generate respiratory rhythm in mammals. Science 254:726-729

24. Mendelowitz D, Kunze DL 1991 Identification and dissociation of cardiovascular neurons from the medulla for patch clamp analysis. Neurosci Lett 132:217-221

25. Wang J, Irnaten M, Venkatesan P, Evans C, Baxi S, Mendelowitz D 2002 Synaptic activation of hypoglossal respiratory motorneurons during inspiration in rats. Neurosci Lett 332:195-199

26. Telgkamp P, Ramirez JM 1999 Differential responses of respiratory nuclei to anoxia in rhythmic brain stem slices of mice. J Neurophysiol 82:2163-2170

27. Lieske SP, Thoby-Brisson M, Telgkamp P, Ramirez JM 2000 Reconfiguration of the neural network controlling multiple breathing patterns: eupnea, sighs, and gasps. Nat Neurosci 3:600-607

28. Bamford OS, Schuen JN, Carroll JL 1996 Effect of nicotine exposure on postnatal ventilatory responses to hypoxia and hypercapnia. Respir Physiol 106:1-11

29. Simakajornboon N, Vlasic V, Li H, Sawnani H 2004 Effect of prenatal nicotine exposure on biphasic hypoxic ventilatory response and protein kinase $\mathrm{C}$ expression in caudal brain stem of developing rats. J Appl Physiol 96:2213-2219

30. Lewis KW, Bosque EM 1995 Deficient hypoxia awakening response in infants of smoking mothers: possible relationship to sudden infant death syndrome. J Pediatr 127:691-699

31. Ueda Y, Stick SM, Hall G, Sly PD 1999 Control of breathing in infants born to smoking mothers. J Pediatr 135:226-232

32. Gilbey MP, Jordan D, Richter DW, Spyer KM 1984 Synaptic mechanisms involved in the inspiratory modulation of vagal cardio-inhibitory neurones in the cat. J Physiol 356:65-78 
33. Neff RA, Wang J, Baxi S, Evans C, Mendelowitz D 2003 Respiratory sinus arrhythmia: endogenous activation of nicotinic receptors mediates respiratory modulation of brainstem cardioinhibitory parasympathetic neurons. Circ Res 93:565-572

34. Garrigue S, Bordier P, Jais P, Shah DC, Hocini M, Raherison C, Tunon De Lara M, Haissaguerre M, Clementy J 2002 Benefit of atrial pacing in sleep apnea syndrome. N Engl J Med 346:404-412

35. Tilkian AG, Guilleminault C, Schroeder JS, Lehrman KL, Simmons FB, Dement WC 1977 Sleep-induced apnea syndrome. Prevalence of cardiac arrhythmias and their reversal after tracheostomy. Am J Med 63:348-358

36. Fewell JE, Smith FG, Ng VK, Wong VH, Wang Y 2000 Postnatal age influences the ability of rats to autoresuscitate from hypoxic-induced apnea. Am J Physiol 279:R39-R46
37. Neff RA, Simmens SJ, Evans C, Mendelowitz D 2004 Prenatal nicotine exposurealters central cardiorespiratory responses to hypoxia in rats: implications for sudden infant death syndrome. J Neurosci 24:9261-9268

38. Poets CF, Meny RG, Chobanian MR, Bonofiglo RE 1999 Gasping and other cardiorespiratory patterns during sudden infant deaths. Pediatr Res 45 $350-354$

39. Huang ZG, Wang X, Evans C, Gold A, Bouairi E, Mendelowitz D 2004 Prenatal nicotine exposure alters the types of nicotinic receptors that facilitate excitatory inputs to cardiac vagal neurons. J Neurophysiol 92:2548-2554

40. Buisson B, Bertrand D 2001 Chronic exposure to nicotine upregulates the human (alpha)4(beta)2 nicotinic acetylcholine receptor function. J Neurosci 21:18191829 